\title{
Safety and Utility of Spinal Magnetic Resonance Imaging in Patients with High-Frequency Spinal Cord Stimulators: A Prospective Single-Centre Study
}

\author{
James Manfield ${ }^{\mathrm{a}}$ Richard Bartlett ${ }^{\mathrm{b}} \quad$ Nicholas Park $^{\mathrm{a}}$ \\ a Department of Neurosurgery, Royal Preston Hospital, Lancashire Teaching Hospitals NHS Foundation Trust, \\ Preston, UK; ${ }^{b}$ Department of Radiology, Royal Preston Hospital, Lancashire Teaching Hospitals NHS Foundation \\ Trust, Preston, UK
}

\section{Keywords}

Spinal magnetic resonance imaging $\cdot$ Spinal cord stimulation · High-fequency spinal cord stimulator implantation

\section{Abstract \\ Background: Implanted high-frequency spinal cord stimula- tors at $10 \mathrm{kHz}$ (HF-SCS) have recently acquired conditional approval for magnetic resonance imaging (MRI), including retrospective application to previously implanted devices. Under certain conditions, there are greater specific absorp- tion rate (SAR) scanning restrictions compared to some con- ventional alternatives. This poses technical challenges to ob- tain diagnostic quality imaging. Objectives: To describe our experience with 9 such scans, demonstrating that safe and diagnostically useful images can be obtained despite these restrictions. Methods: We report a prospective single-centre series of 9 scans within a tertiary neuroscience centre, all ob- tained within the required SAR limit of $\leq 0.4 \mathrm{~W} / \mathrm{kg}$, and de- scribe the scanning protocol we have developed. We further}

illustrate this with 2 representative patient cases. Results: The imaging studies were well tolerated without complication. In all cases, the imaging quality was sufficient for the reporting neuroradiologist to answer the clinical question posed. Conclusion: Despite technical challenges, MRI is feasible, safe and diagnostically useful in HF-SCS-implanted patients. We would invite other centres that implant these devices to consider the development of their own scanning protocols to avoid the morbidity and inconvenience of explantation or computed tomography myelography. To our knowledge, this is the first reported study of MRI in HF-SCSimplanted patients achieving the requisite SAR limit of $\leq 0.4$ $\mathrm{W} / \mathrm{kg}$.

(C) 2019 The Author(s)

Published by S. Karger AG, Basel

\section{Introduction}

Our centre has recently made high-frequency spinal cord stimulators at $10 \mathrm{kHz}$ (HF-SCS) the implant of first choice, over conventional alternatives, following the publi-

\begin{tabular}{ll}
\hline KARGER & $\begin{array}{l}\text { ๑ } 2019 \text { The Author(s) } \\
\text { Published by S. Karger AG, Basel }\end{array}$ \\
$\begin{array}{l}\text { E-Mail karger@karger.com } \\
\text { Open access }\end{array}$ \\
wwww.karger.com/sfn & $\begin{array}{l}\text { This article is licensed under the Creative Commons Attribution 4.0 } \\
\text { International License (CC BY) (http://www.karger.com/Services/ } \\
\text { OpenAccessLicense). Usage, derivative works and distribution are } \\
\text { permitted provided that proper credit is given to the author and the } \\
\text { original publisher. }\end{array}$
\end{tabular}

James Manfield, MBBS, MSc, MRCS

Department of Neurosurgery, Royal Preston Hospital Lancashire Teaching Hospitals NHS Foundation Trust Sharoe Green Lane, Fulwood, Preston PR2 9HT (UK) E-Mail jhrmanfield@ doctors.org.uk 
Table 1. Lumbar T2-weighted MRI scanning parameters

\begin{tabular}{lll}
\hline Parameter & Routine imaging & With Nevro ${ }^{1}$ HF10 \\
\hline Repetition time & 3,692 & 2,903 \\
Time to echo & 120 & 120 \\
Number of excitations & 2 & 1 \\
Bandwidth & 154 & 367.1 \\
Reconstruction matrix & 512 & 512 \\
Sense & no & 1.4 \\
Voxel & $0.9 \times 1.209 \times 4$ & $0.9 \times 1.16 \times 3$ \\
Slice thickness & $4 \mathrm{~mm}$ & $4 \mathrm{~mm}$ \\
Gap & $0.4 \mathrm{~mm}$ & $0.3 \mathrm{~mm}$ \\
Field of view & AT $160 \times$ FH 311 $\times$ LR 66 & AT $200 \times$ FH 371 $\times$ LR 56 \\
Time & $3.30 \mathrm{~s}$ & $0.58 \mathrm{~s}$ \\
\hline
\end{tabular}

Example MRI scanning parameters for lumbar T2-weighted sagittal sequences (routine vs. HF-SCS devices in situ) using our institution's 1.5-T Philip Ingenia MRI Scanner (Philips Corporation, Amsterdam, the Netherlands). ${ }^{1}$ Nevro Corp., Redwood City, CA, USA.

cation of data demonstrating superior efficacy [1]. We have now implanted in excess of 100 HF-SCS devices (Nevro Senza systems; Nevro Corp., Redwood City, CA, USA) for various indications, predominantly failed back surgery syndrome. One traditional downside of these implants was the lack of magnetic resonance imaging (MRI) compatibility, although they have recently acquired conditional approval for full-body MRI, including retrospective application to previously implanted devices [2]. This is a significant advance given it has been estimated that around $82-85 \%$ of SCS-implanted patients will be expected to warrant one or more MRI scans within 5 years of implantation [3]. Given up to 50,000 such devices are implanted each year for various indications, this represents a sizeable clinical need [4].

In common with other implantable devices, there remain several technical MRI challenges which principally stem from specific absorption rate (SAR) scanning restrictions. For an implanted HF-SCS device, when imaging any portion of the spine (or any other structure approximately between the mouth and knee) the wholebody average SAR must not exceed $0.4 \mathrm{~W} / \mathrm{kg}$ with a maximum active scan time of 30 min per study [2]. This is materially below the $2 \mathrm{~W} / \mathrm{kg}$ limit permitted by other lower-frequency stimulator systems (e.g., Medtronic SureScan devices [Medtronic plc, Minneapolis, MN, USA]) and can be challenging to achieve without compromising diagnostic imaging quality. There are several reports in the literature of successful MRI scans with reduced SARs [5-7], with the lowest reported average SAR being 0.74 $\mathrm{W} / \mathrm{kg}$ [7]. However, there have hitherto been no case series described achieving the limit of $0.4 \mathrm{~W} / \mathrm{kg}$ required for
HF-SCS devices. We have now performed 9 scans in 8 patients with HF-SCS devices in situ. These scans have produced diagnostic quality images that have both identified and excluded significant pathological findings, which we describe here and illustrate with 2 case reports.

\section{Methods}

After developing spinal symptoms indicating imaging, 8 patients with implanted HF-SCS percutaneous leads were prospectively investigated by MRI scanning in our tertiary neuroscience centre during 2018. MRI was performed by our MRI radiographers using a particular adapted scanning protocol on our institution's Philips Ingenia 1.5-T MRI scanner (Philips Corporation, Amsterdam, the Netherlands). Our scanning protocol has evolved with experience, and we include our current parameters for sagittal T2weighted lumbar sequences, alongside those for conventional, i.e. non-neuromodulation, scans as a comparison (Table 1). A company representative was present for the initial cases to ensure the implant's compatibility and check impedances to exclude lead integrity deficiency or disconnection. Following training, our inhouse team can now perform these checks independently. A neuroradiologist and/or surgeon were also present to review the images obtained and assess the requirement for further sequences. An MRI physicist attended for some initial scans to aid the development of our reduced SAR scanning protocol.

\section{Results}

In all 9 cases (in 8 patients), the scan was well tolerated with none having to be abandoned due to patient discomfort. None of the patients developed new symptomatol- 
Table 2. Patient and MRI details

\begin{tabular}{|c|c|c|c|c|}
\hline Patient & $\begin{array}{l}\text { Location of } \\
\text { stimulator } \\
\text { wires }\end{array}$ & $\begin{array}{l}\text { Extent of MRI } \\
\text { scan }\end{array}$ & Pathology identified or excluded & $\begin{array}{l}\text { Therapeutic conse- } \\
\text { quences (where appli- } \\
\text { cable) }\end{array}$ \\
\hline 1 & cervical & whole spine $e^{1}$ & $\begin{array}{l}\text { spinal cord, foraminal and cauda equina } \\
\text { compression excluded }\end{array}$ & \\
\hline 2 & thoracic & lumbar spine & $\begin{array}{l}\text { neural compression following } \mathrm{L}_{5} / \mathrm{S}_{1} \\
\text { decompression excluded }\end{array}$ & \\
\hline 3 & thoracic & whole spine & new $\mathrm{L}_{5} / \mathrm{S}_{1}$ right lateral disc extrusion & microdiscectomy \\
\hline 4 & thoracic & whole spine & $\begin{array}{l}\text { spinal cord and cauda equina } \\
\text { compression excluded }\end{array}$ & \\
\hline 5 & thoracic & whole spine & $\begin{array}{l}\text { spinal cord and cauda equina } \\
\text { compression excluded }\end{array}$ & \\
\hline 6 & thoracic & whole spine & $\begin{array}{l}\text { spinal cord and cauda equina } \\
\text { compression excluded }\end{array}$ & \\
\hline 7 & thoracic & cervical spine & multilevel cervical stenosis & $\begin{array}{l}\text { Patient offered and } \\
\text { considering decom- } \\
\text { pressive surgery }\end{array}$ \\
\hline 8 & thoracic & brain & cerebral infarction excluded & \\
\hline 8 & thoracic & whole spine & $\begin{array}{l}\text { spinal cord and radicular compression } \\
\text { excluded; mild cervical stenosis } \\
\text { identified }\end{array}$ & \\
\hline
\end{tabular}

\footnotetext{
${ }^{1}$ Our usual protocol for spinal MRI is to obtain T2-weighted sagittal views of the whole spine with axial images through the clinically/pathologically relevant regions.
}

ogy nor was any stimulator dysfunction identified after MRI scanning. An MRI was planned for a ninth patient but due to unsatisfactory impedance checks, suggesting either a lead not connected to the implantable pulse generator or a lead integrity deficiency, a decision was made not to proceed with the scan, and an alternative modality (CT myelography) was employed.

All 9 images were of sufficient quality for the reporting neuroradiologist to answer the clinical question being asked. Details of the 8 patients who underwent scanning and their imaging are summarised in Table 2 below.

\section{Case Report 1}

A 64-year-old male (patient 1) underwent cervical HF-SCS implantation for uncontrolled neuropathic left-sided arm pain in 2015. Initially, pain relief was excellent until he suffered a fall 18 months later. Following the incident, efficacy was lost which was refractory to reprogramming. The patient also developed left leg weakness and incoordination. Plain radiographs excluded any obvious lead migration or bony injury, hence an MRI scan was performed to exclude a spinal aetiology for his symptoms.
As can be seen from the above images (Fig. 1), the artefact from the epidural leads is fairly minimal, and the patency of the neural foramina as well as the cord signal can be evaluated. On this basis, significant spinal pathology as the cause of his leg symptoms could be excluded, and these did slowly improve over time.

\section{Case Report 2}

A 46-year-old male police officer (patient 3) underwent an HF-SCS implant in 2015 for failed back surgery syndrome, comprising back, leg and bilateral foot pain. He favoured the HF-SCS device as he is required to drive for his occupational duties. The patient had a good outcome, averaging $70 \%$ pain relief overall for over 2 years until he developed an exacerbation of right foot pain from $3 / 10$ to $10 / 10$ on the visual analogue scale, which was refractory to re-programming. An MRI scan was accordingly obtained.

The MRI scan revealed neural compression from prolapse of the $\mathrm{L}_{5} / \mathrm{S}_{1}$ disc (Fig. 2). The patient underwent an uncomplicated right-sided microdiscectomy following which he made a good recovery with symptomatic resolution. 
Fig. 1. a Sagittal T2-weighted MRI of the cervicothoracic spine for patient 1 . b Axial T2-weighted image at level $\mathrm{C}_{4 / 5}$, corresponding to the yellow line on the sagittal image above. The epidural lead is apparent. c Anterior-posterior radiograph of the cervical spine showing the leads in situ without obvious fracture or migration.
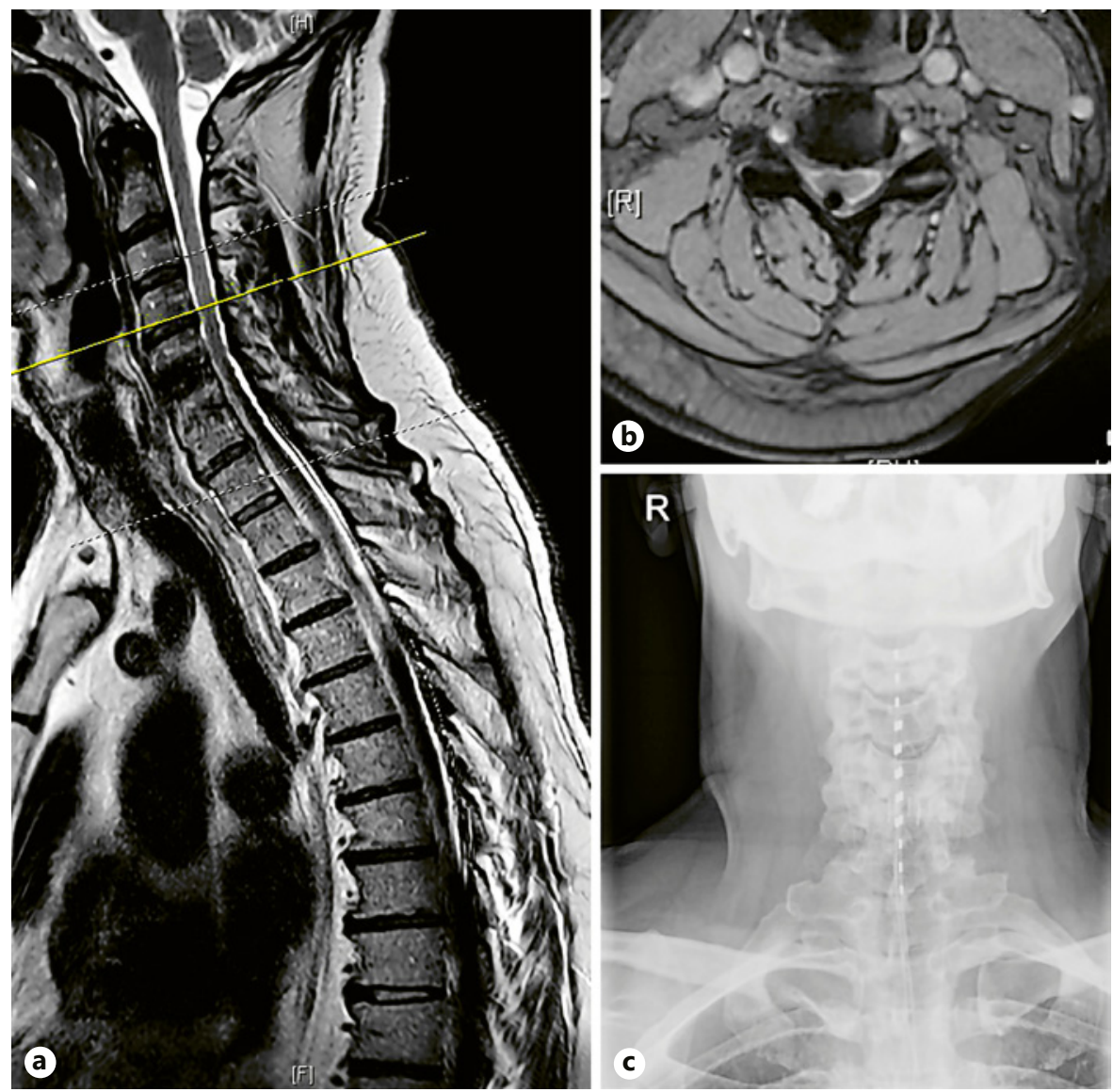

\section{Discussion}

Until 2017, MRI in patients with HF-SCS implants was limited to head and extremity scans. Full-body MRI conditionality has now been acquired for all patients with percutaneous leads in situ, including those with previous implants. Presently, the minority of patients with surgically implanted paddle leads may only undergo head and extremity imaging [2]. In essence, this is due to concerns that the interaction of magnetic, radiofrequency and gradient fields with the ferromagnetic components of the stimulator apparatus might lead to equipment displacement, current induction or heating with consequent tissue damage [8].

In this series, 4 patients were scanned as an emergency, which prior to 2017 would have necessitated either surgical explantation of the stimulator or alternative investigation, e.g. CT myelography. The latter is an invasive procedure which carries small risks of significant complications including seizures and contrast reaction [9], does not image the spinal cord itself, entails ionising radiation expo- sure and is not routinely available out of hours at our centre. Explantation is a surgical procedure and thus exposes the patient to considerable morbidity and inconvenience. In view of these factors, as well as its status as the gold standard imaging modality, MRI is now our first-line investigation in HF-SCS patients requiring spinal imaging.

There has inevitably been a learning curve with the development and refinement of our local scanning protocol to obtain adequate images within the requisite SAR limit, which initially required significant input from an MRI physicist. The scanning process for these patients is more labour intensive as it requires the presence of trained personnel to check lead impedances as well as a clinician (radiologist and/or spinal surgeon) to review the images in real time to guide the radiographer as to which further sequences are required. Typically, this entails evaluating the initial T2-weighted sagittal images and tailoring axial cuts to just the region(s) of pathological significance. The lengthened repetition time also means that the overall scan takes longer than usual, although cannot exceed 30 min of active scan time without a 60 -min gap. As a result, 
2

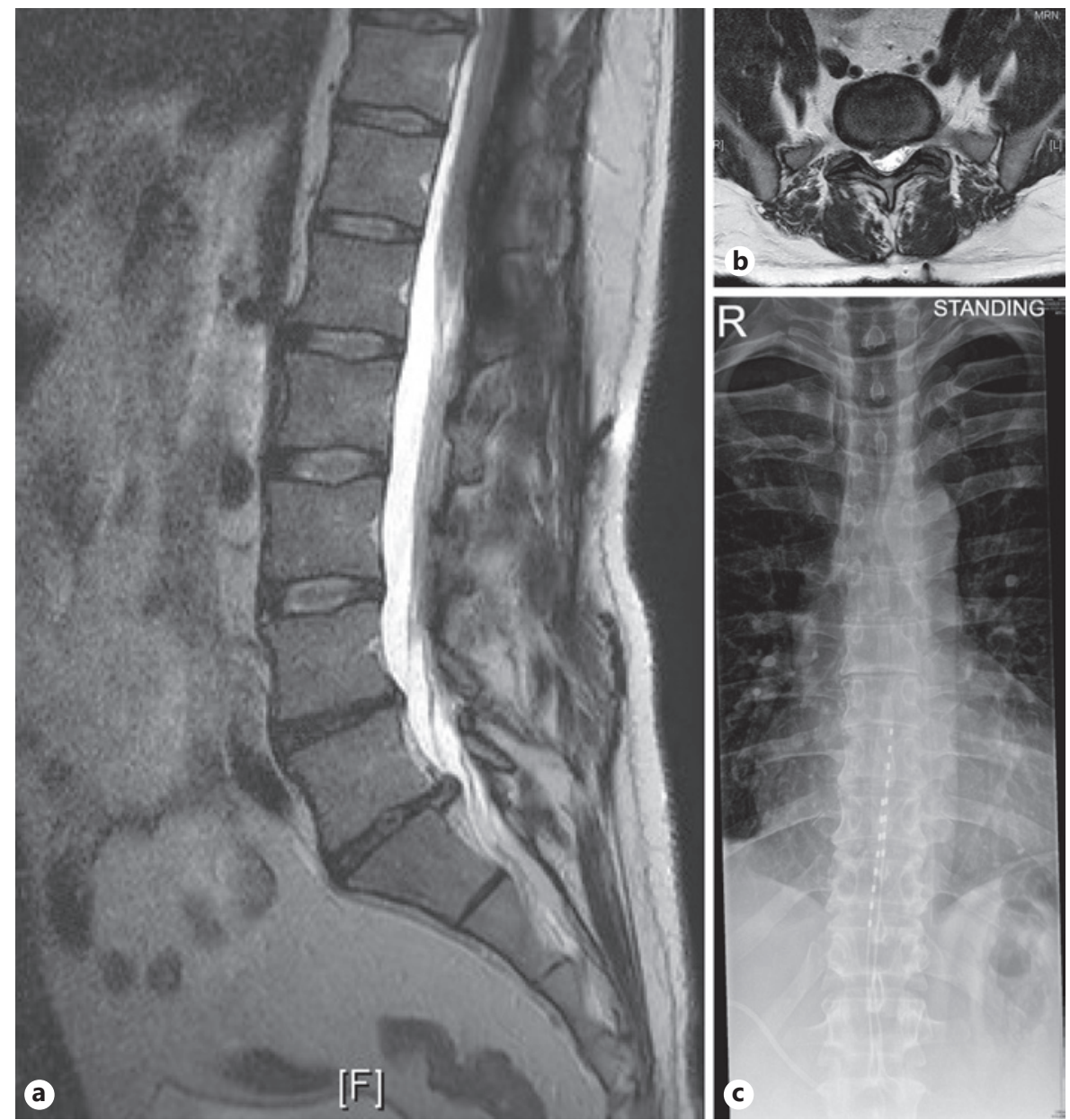

Fig. 2. a Sagittal T2-weighted MRI through the lumbar spine revealing prolapsed intervertebral disc at $\mathrm{L}_{5} / \mathrm{S}_{1}$. b Axial T2-weighted image at $\mathrm{L}_{5} / \mathrm{S}_{1}$ demonstrating the disc prolapse and resultant neural compression. c Anterior-posterior radiograph demonstrating satisfactory positioning of the spinal cord stimulation leads.

Fig. 3. a, b T2-weighted sagittal (a, whole spine) and axial (b, $\mathrm{T}_{10 / 11}$ ) level MRI sequences of patient 2 demonstrating signal drop-out imaging artefact from epidural leads.

\section{3}
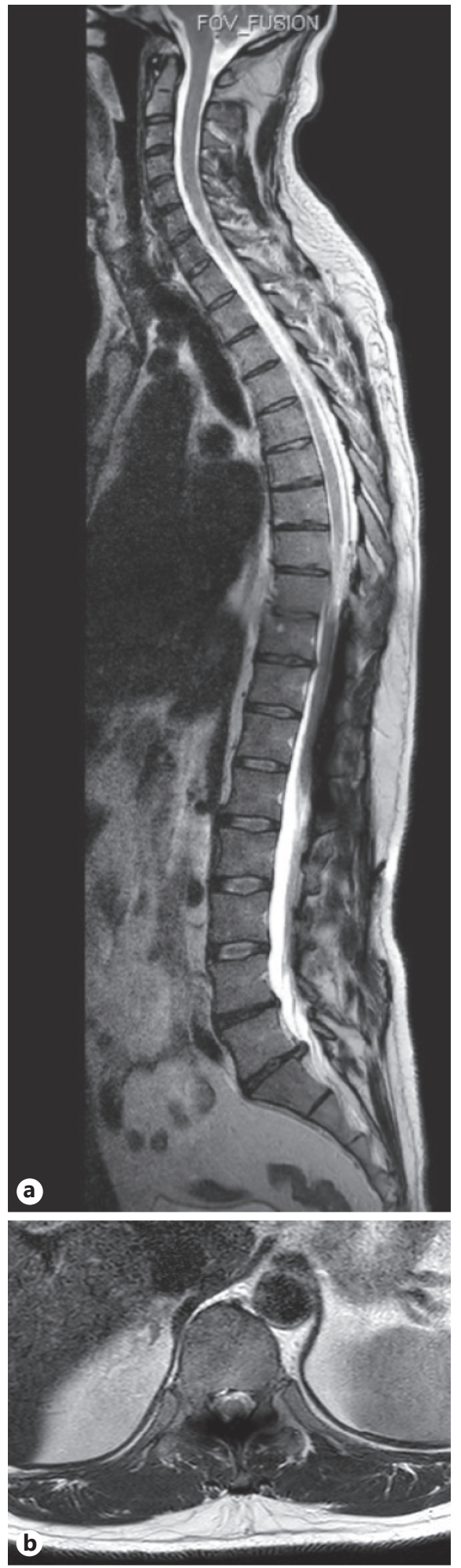
patients must be reasonably compliant and not claustrophobic to tolerate the scan. It is our practice to carefully counsel the patient about what to expect and endeavour to optimise analgesia prior to imaging.

Artefacts causing signal drop-out in close vicinity of the electrodes do occur but have never caused a diagnostic problem in our experience (Fig. 3). It remains possible that a subtle lesion in the region of the electrodes could be missed. Based on this series, we have been able to exclude spinal cord and cauda equina compression with confidence. Spinal cord signal change and neuroforaminal compromise can also be identified even adjacent to an electrode.

\section{Conclusion}

To our knowledge, we have presented the first case series of spinal MRI in patients harbouring HF-SCS at $10 \mathrm{kHz}$ stimulators, with imaging performed at a SAR level $(<0.4 \mathrm{~W} / \mathrm{kg})$ not previously described in the literature. This demonstrates that with a specifically adapted reduced SAR protocol, diagnostic quality images can be safely achieved and are well tolerated by appropriate patients. As a consequence, since 2017, we have not had to explant any patient's stimulator to facilitate imaging. On only one occasion has CT myelography been necessary (due to either a lead not connected to the implantable pulse generator or a lead integrity deficiency). We invite other centres that implant these HF-SCS stimulators to consider developing their own MRI scanning protocol as it remains the gold standard investigation to assess the neuraxis and avoid the morbidity and inconvenience associated with myelography or explantation.

\section{Statement of Ethics}

As MRI scanning is FDA approved and was performed as part of routine clinical care, no specific IRB/ethics committee approval was needed. All patients consented to undergo MRI scanning. Patient consent for publication was not required since no identifiable data are included in the paper.

\section{Disclosure Statement}

Mr. Park has previously received sponsorship and consultancy fees from Nevro, and sponsorship from Abbott and Medtronic all outside the submitted work. Mr. Manfield and Dr. Bartlett have no conflict. All authors have no personal, financial or institutional interest in any of the materials or devices described in this article.

\section{Funding Sources}

No funding was received for the study.

\section{Author Contributions}

James Manfield and Nicholas Park co-conceived the study and drafted the manuscript. Richard Bartlett proved radiological expertise, for images and scanning protocols, and reviewed the manuscript.

\section{References}

1 Kapural L, Yu C, Doust MW, Gliner BE, Vallejo R, Sitzman BT, et al. Novel $10-\mathrm{kHz}$ high-frequency therapy (HF10 therapy) is superior to traditional low-frequency spinal cord stimulation for the treatment of chronic back and leg pain. The SENZA-RCT Randomized Controlled Trial. Anesthesiology. 2015;123(4):851-60.

2 Nevro: MRI guidelines for the SENZA and SENZA II systems [cited 2018 Oct 22]. Available from: https://www.nevro.com/English/ Physicians/manuals/default.aspx

3 Desai MJ, Hargens LM, Breitenfeldt MD, Doth AH, Ryan MP, Gunnarsson C, et al. The rate of magnetic resonance imaging in patients with spinal cord stimulation. Spine. 2015 May;40(9):E531-7.
4 Mekhail NA, Cheng J, Narouze S, Kapural L, Mekhail MN, Deer T. Clinical applications of neurostimulation: forty years later. Pain Pract. 2010 Mar-Apr;10(2):103-12.

5 De Andres J, Valía JC, Cerda-Olmedo G, Quiroz C, Villanueva V, Martinez-Sanjuan V, et al. Magnetic resonance imaging in patients with spinal neurostimulation systems. Anesthesiology. 2007 Apr;106(4):779-86.

6 De Andres J, Martinez-Sanjuan V, FabregatCid G, Asensio-Samper JM, Sanchis-Lopez N, Villanueva-Perez V. MRI-compatible spinal cord stimulator device and related changes in patient safety and imaging artifacts. Pain Med. 2014 Oct;15(10):1815-9.
7 Mutter UM, Bellut D, Porchet F, Schuknecht B. Spinal magnetic resonance imaging with reduced specific absorption rate in patients harbouring a spinal cord stimulation device - A single-centre prospective study analysing safety, tolerability and image quality. Acta Neurochir (Wien). 2013 Dec;155(12):2327-32.

8 Rubino S, Adepoju A, Kumar V, Prusik J, Murphy N, Owusu-Sarpong S, et al. MRI Conditionality in patients with spinal cord stimulation devices. Stereotact Funct Neurosurg. 2016;94(4):254-8.

9 Sandow BA, Donnal JF. Myelography complications and current practice patterns. AJR Am J Roentgenol. 2005 Sep;185(3):768-71. 